\title{
Experimental investigation of liquid jet injection into Mach 6 hypersonic crossflow
}

\author{
J. Beloki Perurena $\cdot$ C. O. Asma \\ R. Theunissen $\cdot$ O. Chazot
}

Received: 29 February 2008/Revised: 28 August 2008/Accepted: 29 August 2008/Published online: 17 September 2008

(C) Springer-Verlag 2008

\begin{abstract}
The injection of a liquid jet into a crossing Mach 6 air flow is investigated. Experiments were conducted on a sharp leading edge flat plate with flush mounted injectors. Water jets were introduced through different nozzle shapes at relevant jet-to-air momentumflux ratios. Sufficient temporal resolution to capture small scale effects was obtained by high-speed recording, while directional illumination allowed variation in field of view. Shock pattern and flow topology were visualized by Schlieren-technique. Correlations are proposed on relating water jet penetration height and lateral extension with the injection ratio and orifice diameter for circular injector jets. Penetration height and lateral extension are compared for different injector shapes at relevant jet-to-air momentum-
\end{abstract}

J. Beloki Perurena - C. O. Asma - R. Theunissen .

O. Chazot

von Karman Institute for Fluid Dynamics,

1640 Rhode-Saint-Genèse, Belgium

e-mail: chazot@vki.ac.be

Present Address:

J. Beloki Perurena $(\square)$

Shock Wave Laboratory, RWTH Aachen University,

52056 Aachen, Germany

e-mail: beloki@vki.ac.be; beloki@swl.rwth-aachen.de

C. O. Asma

Department of Flow, Heat and Combustion Mechanics,

Ghent University, 9000 Ghent, Belgium

e-mail: asma@vki.ac.be

R. Theunissen

Faculty of Aerospace Engineering,

Delft University of Technology, 2629 HS Delft,

The Netherlands

e-mail: theuniss@vki.ac.be flux ratios showing that penetration height and lateral extension decrease and increase, respectively, with injector's aspect ratio. Probability density function analysis has shown that the mixing of the jet with the crossflow is completed at a distance of $x / d_{j} \sim 40$, independent of the momentum-flux ratio. Mean velocity profiles related with the liquid jet have been extracted by means of an ensemble correlation PIV algorithm. Finally, frequency analyses of the jet breakup and fluctuating shock pattern are performed using a Fast Fourier algorithm and characteristic Strouhal numbers of $S t=0.18$ for the liquid jet breakup and of $S t=0.011$ for the separation shock fluctuation are obtained.

\section{List of symbols}

$A_{j} \quad$ area of injected jet orifice $\left(\mathrm{m}^{2}\right)$

AR aspect ratio (-)

$d_{j} \quad$ diameter of injected round jet orifice (m)

$M \quad$ Mach number (-)

$P_{0} \quad$ stagnation pressure (Bar)

$q \quad$ momentum-flux ratio or injection ratio (-)

Re Reynolds number (-)

SN signal-to-noise ratio (-)

St Strouhal number (-)

$T_{\infty} \quad$ temperature of free stream air $(\mathrm{K})$

$T_{0} \quad$ stagnation temperature $(\mathrm{K})$

$U_{\infty} \quad$ velocity of free stream air $(\mathrm{m} / \mathrm{s})$

$U_{j} \quad$ velocity of injected jet $(\mathrm{m} / \mathrm{s})$

$\rho_{\infty} \quad$ density of free stream air $\left(\mathrm{kg} / \mathrm{m}^{3}\right)$

$\rho_{j} \quad$ density of injected jet $\left(\mathrm{kg} / \mathrm{m}^{3}\right)$

$h(x) \quad$ penetration height $(\mathrm{m})$

$w(x) \quad$ lateral extension (m) 


\section{Introduction}

The flow topology related with liquid jet injection into supersonic and hypersonic crossflow is currently of large interest. Applications related with this topic cover a wide range from thrust vectoring of high speed vehicles to fuel injection in supersonic combustion ramjet engines and transpiration cooling of re-entry vehicles. The breakup process of a liquid jet injected into a crossing hypersonic flow is, however, considerably complex. A simplified schematic of the involved flow topology is presented in Fig. 1. Upstream of the bow shock, which is located in front of the injected liquid jet, the accompanying adverse pressure gradient causes the boundary layer to detach, yielding a separation shock. Separation and bow shocks interact, giving rise to alterations in pressure distribution, which in turn modify the bow shock angle. The latter induces a shift in separation point and inclination of separation shock, creating a further change in bow shock angle and jet flow. The resolving chain in events is perceived macroscopically as a continuous vibration of the shock system, which is also called whipping phenomena. The appearance of a separation shock further forces the liquid jet to mix with the air upstream of the injection under subsonic conditions. Exactly this zone is of importance in cases involving combustion due to its flame-holding capability (Gruber et al. 1996; Yakar and Hanson 1998). After injection, the jet is deflected in a manner dependent on the momentum-flux ratio (Schetz et al. 1980). Downstream of liquid injection point, the surface of the deflected jet is subject to wavelike disturbances (Sallam et al. 2004). According to Schetz et al. (1980) these are most likely attributed to Kelvin-Helmholtz instabilities as a result of the acceleration of a greater density fluid by a high-speed fluid flow of lower density. As reported by Less and Schetz (1986), these waves grow in amplitude, wavelength and speed on the surface of the jet, yielding the primary breakup in the form of clump detachment along the outer portion of the jet (Fig. 1). This abrupt clump detachment

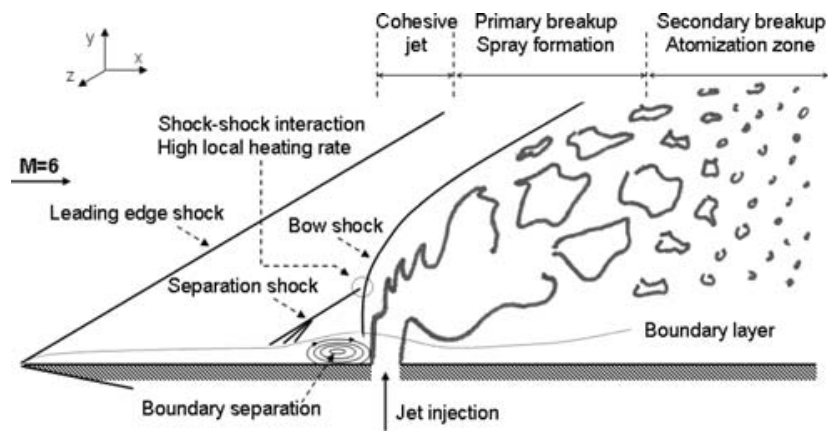

Fig. 1 Schematic of liquid jet injection into hypersonic crossflow can be promoted by the bow shock-separation shock interaction and the increasing pressure and heat flux associated with it (Delery 1989). In the secondary breakup zone, the formed spray decomposes into ever smaller droplets under action of aerodynamic forces of the crossflow until a critical droplet radius is reached. The latter is attained when surface tension force in the droplet surpasses the aerodynamic forces.

In this interaction between the crossflow and the jet, well-known characteristic vortical structures appear:

(a) Transverse vortices on the boundaries of the jet, named jet-shear-layer vortices, stemming from Kelvin-Helmholtz instabilities.

(b) Counter rotating vortex pair or kidney vortices, produced by folding of the vortex ring, which is a downstream manifestation of the vorticity arising from the injector's sidewall boundary layers. This vortex system has been extensively reported in many studies (e.g. Haven and Kurosaka 1997; Cortelezzi and Karagozian 2001). Haven and Kurosaka (1997) demonstrated that the kidney vortices are generated from the boundary layer of the injector's sidewalls and the injector geometry has a strong influence on the near field character of the kidney vortices.

(c) Horseshoe vortices, due to the reverse flow upstream of the jet resulting from the blockage of the main flow by the jet (Krothapalli et al. 1989).

(d) Wake vortices downstream of the jet injection. Fric and Roshko (1994) discovered that these vortices are generated from the vorticity of the boundary layer of the bottom wall and propagate vertically up to the jet.

A schematic representation of the above phenomena according to Fric and Roshko (1994) is depicted in Fig. 2.

Many studies have been devoted to the liquid jet's penetration height into supersonic crossflow in view of its

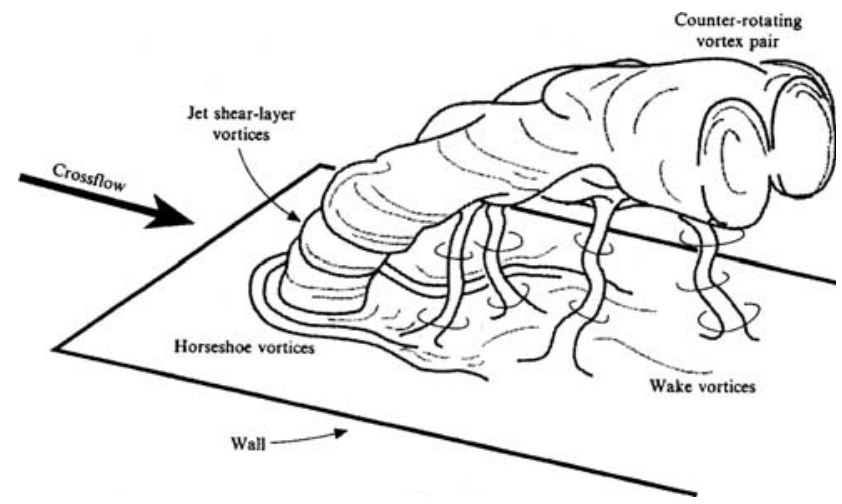

Fig. 2 Characteristic vortical structures in jet injection into crossflow topology (Fric and Roshko 1994) 
important relation between mixing performance and combustion efficiency. Kolpin et al. (1968) proposed a simple correlation between the penetration height and the momentum-flux ratio based on experimental data. Joshi and Schetz (1975) arrived to similar conclusions assessing the injector geometry dependence on penetration height. They also show that lateral dimension of the jet depends more on the lateral extension of the injector than the momentum-flux ratio. Lin et al. (2002a) have proposed one of the newest correlations based on shadowgraph measurements, in which, alike the previous studies, the penetration height relates to the momentum-flux ratio and downstream distance. Lin et al. (2004) obtained a similar correlation based on PDPA measurements, differing the constants and powers of the correlation. Lin et al. (2002b) and Ghenai et al. (2005) demonstrated liquid jet aeration, presenting a two-phase flow comprising air and liquid, to increase the jet penetration height due to an increase of momentum-flux ratio between the jet and crossflow. In a similar manner, Muraggapan et al. (2005) showed, for a supersonic gas injection study, that forced injection provides higher penetration of the jet into the free stream in comparison to baseline jets due to the periodic appearance of large-scale eddies on the jet/free stream interface. Further research has been devoted to other important parameters such as the breakup and structure of these jets. Regarding the breakup, Less and Schetz (1986) discovered that the jet column fractures shortly after the sonic point behind the curved bow shock, which was verified by Li and Karagozian (1992). On the topic of the structure of the jet, Lin et al. (2004) discovered that centerline distribution profiles of droplet plume properties in the freestream direction can be normalized by the penetration height of each spray to obtain universal curves in the regions where the liquid atomization process is complete

The effects of liquid properties such as viscosity and surface tension have been studied by Reichenbach and Horn (1971) concluding that these properties do not affect the penetration height for very high crossflow velocities. Nejad and Schetz (1984) on the other hand discovered that by decreasing the surface tension both the wavelength and wave amplitude increase, intensifying the process of breakup whereas wavelength and amplitude decrease and increase, respectively, with viscosity. According to Schetz et al. (1980) the momentum-flux ratio is also an important parameter of the flow topology revealing the characterization of high injection rate jets $(q>6)$ to be well defined by a jet body while low injection rate jets $(q<1)$ breakup immediately upon injection and vibrate continuously.

Most of the mentioned research is confined to supersonic conditions whereas scramjet engines could also operate in the hypersonic regime. Bibliography regarding hypersonic flow is not plenty and not much data is available. Catton et al. (1968) arrived for hypersonic flows to similar conclusions as Kolpin et al. (1968) for supersonic flows and showed that the penetration height is a function of the momentum-flux ratio, the angle of injection and the orifice geometry. Hinson et al. (1970) attested that variation in inclination of the water injection from a perpendicular to a lower angle reduces the penetration height and the lateral extension. As no profuse data is available regarding hypersonic crossflow, the present study presents results that give a better insight of the flow topology of liquid jet injection into hypersonic crossflow. Penetration height, lateral extension, mixing, velocity of the injected jet and frequency of jet breakup and shock pattern are experimentally studied for water injection through implementation of diverse image processing techniques. Extracted penetration height and lateral extension for different injector shapes are compared with literature. Combined with the obtained data under different test configurations, an expression for the penetration height of jet injection from a circular injector will furthermore be proposed as a function of the injection ratio and the downstream distance.

\section{Experimental setup}

Different experimental conditions have been studied corresponding to variations in momentum-flux ratio, injector shape and tunnel stagnation pressure. Some characteristic examples of the performed tests and the different experimental techniques are summarized in Table 1. Each injector is tested for relevant values of injection ratios $q$, ranging from 1 to 10 . For clarity the expression of $q$ is presented in Eq. 1

$q=\frac{\rho_{j} U_{j}^{2}}{\rho_{\infty} U_{\infty}^{2}}$

\subsection{Wind tunnel facility}

Experiments are carried out in the $\mathrm{H} 3$ hypersonic wind tunnel of the von Karman Institute for fluid dynamics (Fig. 3). Air supplied from a pebble-bed heater is blown down with an axisymmetric nozzle yielding a uniform Mach 6 free jet of $12 \mathrm{~cm}$ in diameter. Stagnation pressure ranges between 20 and 30 bars $( \pm 3 \%)$ with stagnation temperatures in the order of $500 \mathrm{~K}( \pm 4 \%)$. This leads to a range of unit Reynolds number between $R e=15 \times 10^{6}$ and $25 \times 10^{6} / \mathrm{m}$, respectively. Stagnation pressure and temperature are measured, respectively, by a variable reluctance differential pressure transducer and a K-type thermocouple. Optical access is ensured by glass windows installed on the top and both sides of the test section. 
Table 1 Liquid jet and free stream conditions for some test examples

\begin{tabular}{llllllrrr}
\hline Test no & $P_{0}(\mathrm{Bar})$ & $T_{0}(\mathrm{~K})$ & $\rho_{\infty}\left(\mathrm{kg} / \mathrm{m}^{3}\right)$ & $U_{\infty}(\mathrm{m} / \mathrm{s})$ & $\rho_{j}\left(\mathrm{~kg} / \mathrm{m}^{3}\right)$ & $U_{j}(\mathrm{~m} / \mathrm{s})$ & $q$ & Test technique \\
\hline 1 & 20.23 & 505.6 & 0.0724 & 944.39 & 997.93 & 11.38 & 2.0 & Schlieren \\
2 & 20.15 & 508.2 & 0.0718 & 946.82 & 997.83 & 16.06 & 4.0 & Schlieren \\
3 & 20.22 & 510.6 & 0.0717 & 949.05 & 997.78 & 25.43 & 10.0 & Schlieren \\
4 & 20.16 & 502.9 & 0.0725 & 941.87 & 998.06 & 21.40 & 7.1 & Front-lighted \\
5 & 20.25 & 503.4 & 0.0728 & 942.34 & 998.14 & 25.58 & 10.1 & Front-lighted \\
6 & 20.15 & 501.6 & 0.0727 & 940.65 & 997.98 & 16.06 & 4.0 & Front-lighted \\
7 & 20.07 & 513.2 & 0.0708 & 951.46 & 997.93 & 25.46 & 10.1 & Back-lighted \\
8 & 20.12 & 509.6 & 0.0714 & 948.12 & 997.96 & 21.23 & 7.0 & Back-lighted \\
9 & 20.73 & 511.7 & 0.0733 & 950.07 & 997.96 & 8.14 & 1.0 & Back-lighted \\
10 & 20.12 & 508.5 & 0.0716 & 947.10 & 997.96 & 25.37 & 10.0 & Top view \\
11 & 20.68 & 513.2 & 0.0729 & 951.46 & 997.91 & 21.52 & 7.0 & Top view \\
12 & 20.35 & 506.3 & 0.0727 & 945.05 & 997.86 & 11.41 & 2.0 & Top view \\
\hline
\end{tabular}

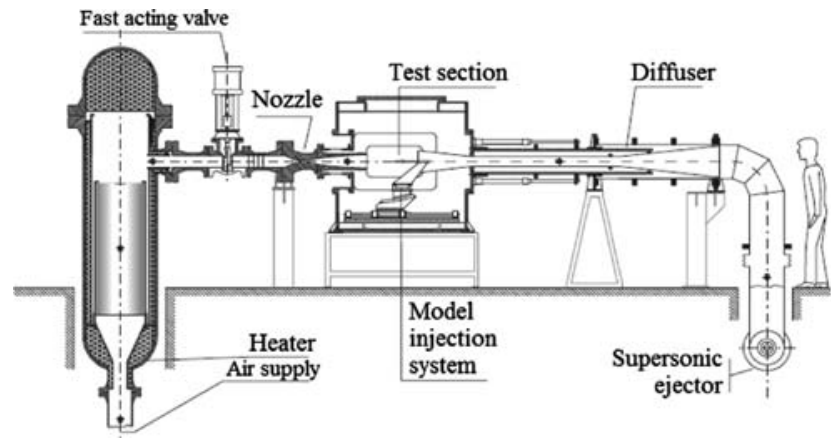

Fig. 3 Schematic of H3 hypersonic wind tunnel

\subsection{Test model}

Water was injected through a flat plate test model of $230 \times 80 \times 15 \mathrm{~mm}$. The injectors were fitted beneath the flat plate while the orifice was flush mounted to the flat surface and positioned $45 \mathrm{~mm}$ downstream of the sharp leading edge. Figure 4 depicts the flat plate mounted in the test section.

Three different geometries of injectors utilized were a streamwise rectangular injector of $0.5 \times 1.6 \mathrm{~mm}^{2}$ (aspect ratio $=1 / 3$ ), a circular injector of $1 \mathrm{~mm}$ diameter (aspect ratio $=1$ ) and a spanwise rectangular injector of $1.6 \times 0.5 \mathrm{~mm}^{2}$ (aspect ratio $=3$ ). The rectangular injectors' edges were rounded in order to obtain more uniform flow over the rectangle. The range of jet Reynolds numbers based on the jet exit diameter $d$ and the exit velocity, varied from $R e=11,000$ for low injection rates up to $R e=28,000$ for the highest injection rate. Figure 5 depicts a detailed view of the three injectors used during the investigation.

Throughout the test-matrix, tap water at room temperature was used as liquid injectant. The water temperature was measured with a $\mathrm{K}$ type thermocouple and monitored

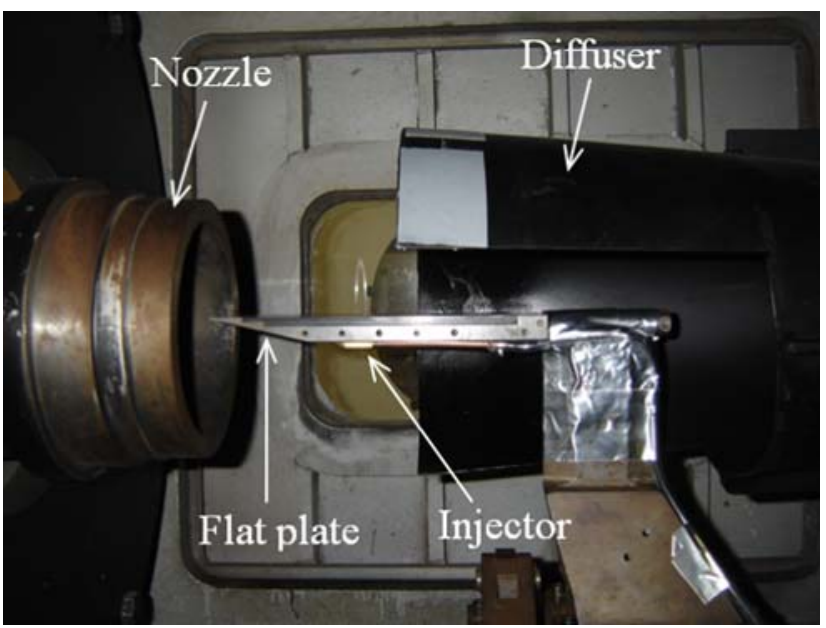

Fig. 4 Flat plate model mounted into test section

by a data acquisition system box. Frequencies of fluctuations of the injected water pressure were observed by a pressure transducer. A rotameter downstream of the first valve fixed the mass flow rate of the water and of the desired momentum-flux ratio. A schematic of the setup is shown in Fig. 6.

\subsection{Flow illumination, recording and visualization}

During all experimental configurations, a Phantom V7.0 digital high speed camera acquired data images. Considering the small time delay between individual recordings, the recording frequency was sufficiently high (maximum $65 \mathrm{kHz}$ ) to capture small-scale features.

Visualization of the flow shock pattern was performed by means of the Schlieren imaging technique (Fig. 7a). Lunartron Electronics Ltd. provided a continuous point source of light, collimated by a first parabolic mirror. Flat mirrors were mounted to pass parallel rays of light through 


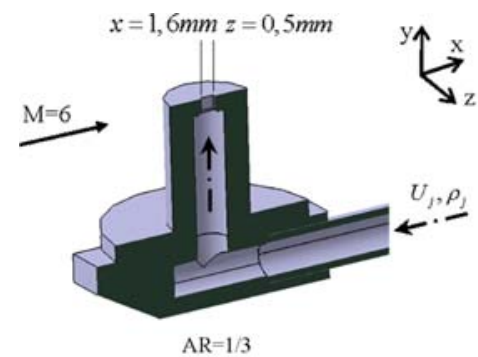

(a)

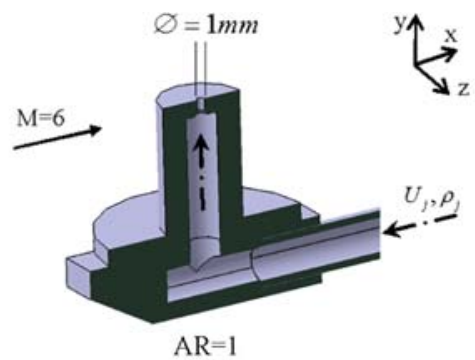

(b)

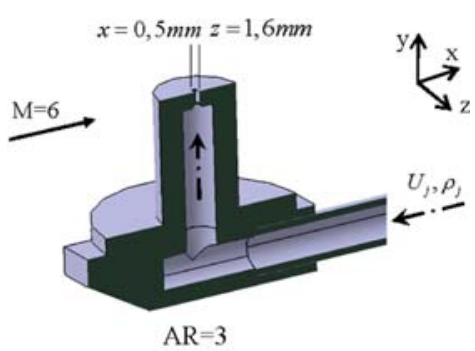

(c)

Fig. 5 a Streamwise rectangular injector, $A R=1 / 3 ; \mathbf{b}$ circular injector, $A R=1$ and $\mathbf{c}$ spanwise rectangular injector, $A R=3$
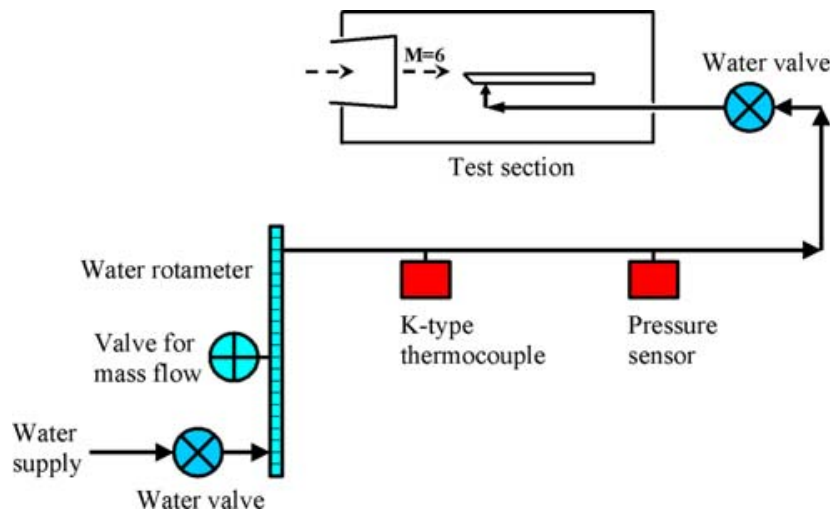

Fig. 6 Experimental setup

the test section and reflect them to a second parabolic mirror. The latter focused the light, thereby reimaging the point towards the camera after interception by an adjustable knife-edge. Optical access was possible up to a distance of $50 \mathrm{~mm}$ downstream of the injection.

A sample image from a Schlieren movie for a $q=10$ circular injector jet case, recorded at a sampling rate of $8,810 \mathrm{~Hz}$ is shown in Fig. 7b. The typical field of view for this setup $\left(704 \times 360\right.$ pixels $\left.^{2}\right)$ corresponded to $168 \times 86 \mathrm{~mm}^{2}$ in object space. Bow shock, separation shock and boundary layer separation are readily distinguishable which allows deduction of the leading edge shock angle. Based on weak disturbance theory the Mach angle " $\sigma$ " is defined by $\sin (\sigma)=M^{-1}$. Presently, the Mach number equaled 6 , yielding a theoretical wave angle of $9.59^{\circ}$. Image analysis returns an angle of approximately $9.89^{\circ}$. The latter verifies the assumption of a weak wave created by the flat plate's leading edge.

To obtain a wide view of the flow field the test section was front-illuminated by two halogen lamps of 1,600 and $1,000 \mathrm{~W}$ (Fig. 8a). Recorded images are used to extract penetration height and to perform the mixing analysis based on probability density functions (PDF). A sample image in case of a circular injector with injection ratio $q=10$, recorded at a sampling rate of $26,143 \mathrm{~Hz}$, is presented in Fig. 8b. The typical field of view for this setup was $512 \times 144$ pixels $^{2}$, corresponding to $213 \times 60 \mathrm{~mm}^{2}$.

An adapted PIV technique was applied for the extraction of velocity data. Rather than recording the scattered light intensities as in conventional PIV systems, shadows constituted the patterns to track. To increase the spatial resolution, the field of view was narrowed to $304 \times 184$ pixels $^{2}$ $\left(32 \times 20 \mathrm{~mm}^{2}\right)$. Sufficient contrast between liquid (shadow) and free stream (illuminated) was obtained through backlight illumination with a halogen lamp of 1,600 W (Fig. 9a). To minimize the degrading influences of spatial variations in intensity during the image analyses, a background image was subtracted beforehand. The background image was based on the ensemble averaged reference images of the illumination, i.e. images without liquid injection.

Figure $9 b$ shows a typical sample of four consecutive frames of back-light illumination with inverted gray-scales. This sample represents liquid injection through the circular injector, with $q=10$, at a recording rate of $63,492 \mathrm{~Hz}$. In the succession of images, the primary breakup and clump detachment on the windward region of the jet are visualized. In the literature, this process is explained as a result of
Fig. 7 a Schlieren test setup; b sample image for a $q=10$ circular injector jet

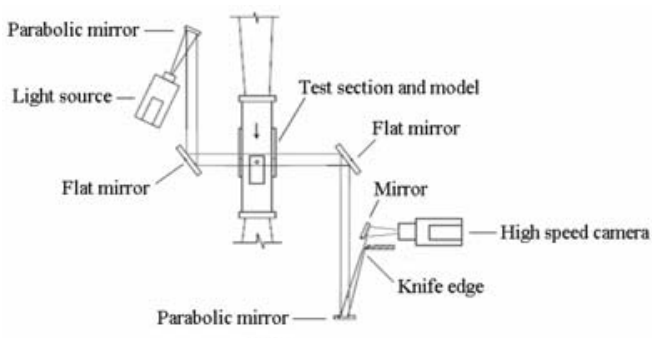

(a)

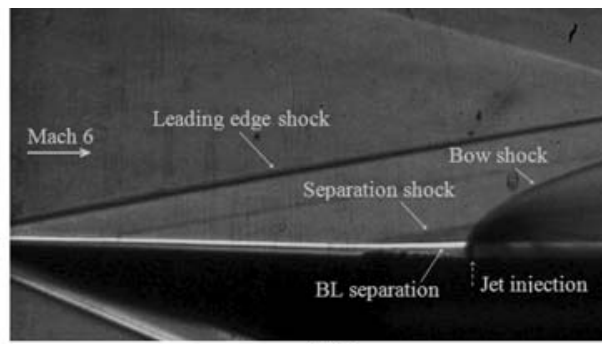

(b) 
Fig. 8 a Front-lighted illumination setup; b sample image for a $q=10$ circular injector jet

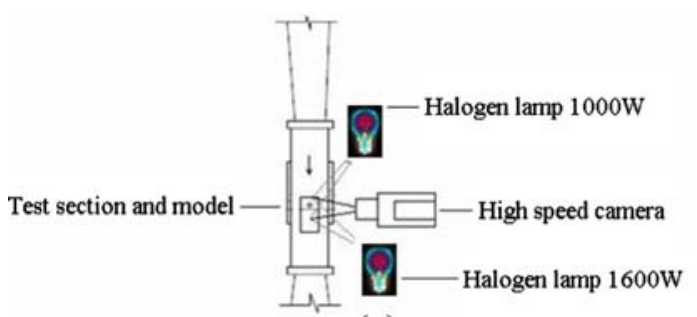

(a)

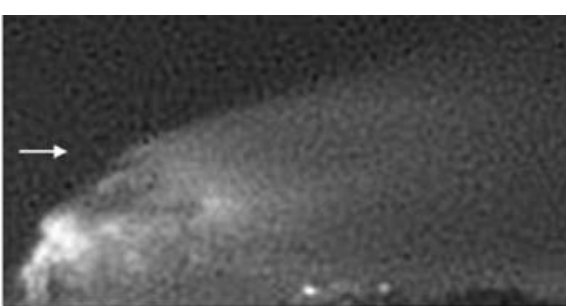

(b)

Fig. 9 a Back-lighted illumination setup; b succession of images with inverted gray-scales

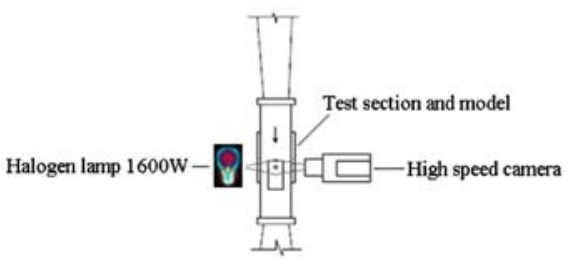

(a)
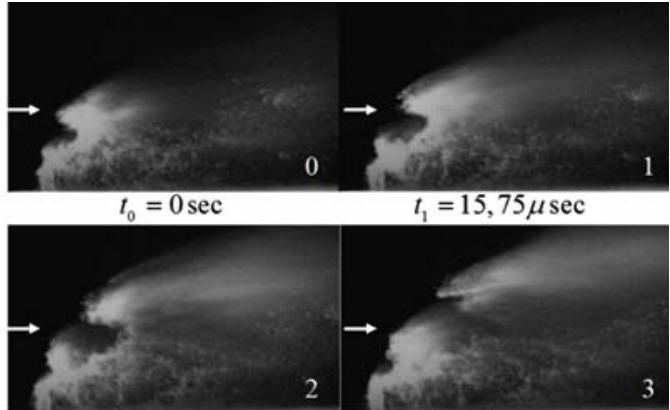

(b) the growing instabilities on the surface of the jet. For the hypersonic conditions of this study, the clump detachment is more violent than in supersonic or subsonic injections. This suggests the shock-shock interaction influence on the stability of the liquid column, yielding to a more abrupt breakup of the jet.

The relation between the clump detachment and the separation shock-bow shock interaction is clearer identified in the high-speed Schlieren recordings. To better visualize this relation, a superposition between a Schlieren image (Fig. 10a) and the close-up view of a jet breakup image, for a $q=10$ circular jet, is shown in Fig. 10b. The image shows that the impingement point of the separation shock on the bow shock coincides with the clump detachment region on the windward region of the jet.

To investigate the spreading of the jet in the lateral extension, top view images were utilized. Appropriate illumination was provided by placing two halogen lamps of 1,600 and 1,000 $\mathrm{W}$ from the side windows while placing the high-speed camera directly above the model (Fig. 11a). Given the experimental conditions and setup, a field of view of $160 \times 184$ pixels $^{2}\left(53 \times 61 \mathrm{~mm}^{2}\right.$ in physical dimensions) was covered. A sample image from a top view movie for a $q=10$ jet through the circular injector, recorded at a sampling rate of $30,075 \mathrm{~Hz}$, is shown in Fig. $11 \mathrm{~b}$.

\section{Image analysis}

Following the experiments, the recorded images are postprocessed to obtain scientific data. The principle tools comprised of in-house developed software (Marangoz et al. 2006).

\subsection{Penetration height}

Measurement of variations in jet's penetration height was obtained from front-light illuminated images (Fig. 12a). To facilitate contour detection of the jet, images were binarized. Pixel intensities exceeding a pre-imposed intensity gradient threshold were set to unity and zero otherwise. The standard deviation over 1,000 binary images was used

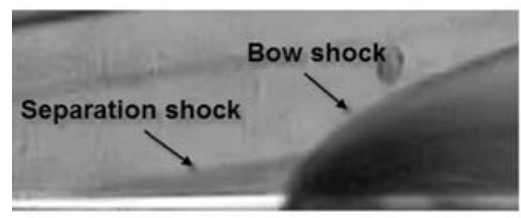

(a)

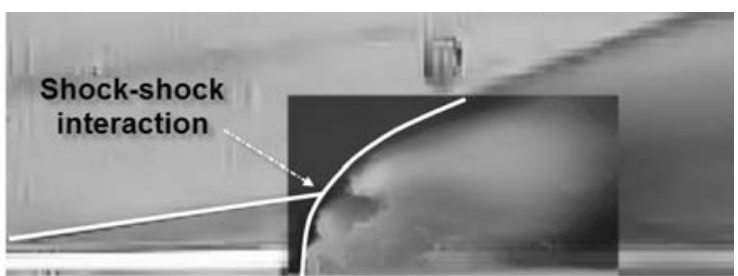

(b)

Fig. 10 a Schlieren image for $q=10$ jet; $\mathbf{b}$ superposition of Schlieren image and close-up view of jet breakup 
Fig. 11 a Top view test setup;

b sample image for a $q=10$ circular injector jet

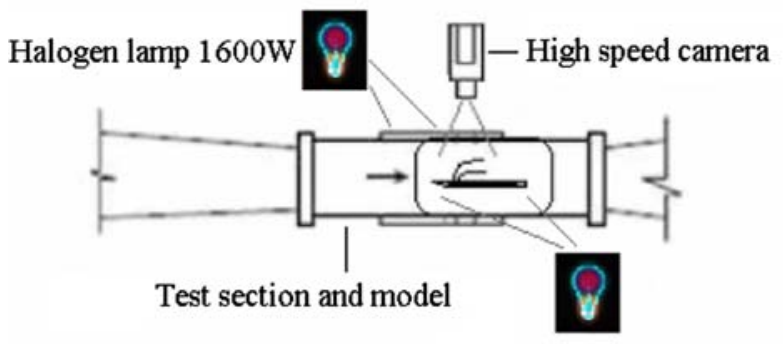

Halogen lamp 1000W

(a)

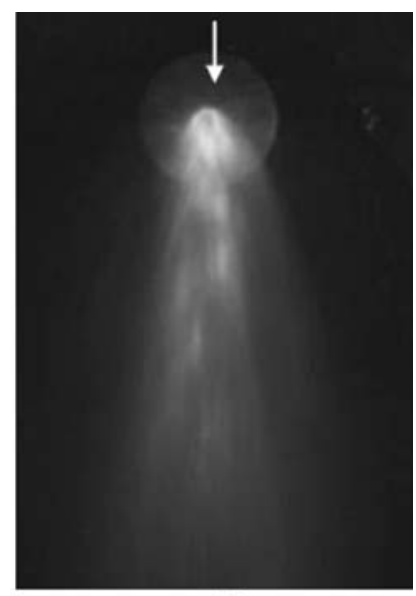

(b) to determine the penetration height. An example of this is shown in Fig. 12b. The measurements are estimated to be accurate within $2 \%$.

The boundary of the circular jet directly exposed to the free-stream for different injection ratios are presented in Fig. 13. Experimental profiles for circular jet injections into supersonic Mach $=1.94$ crossflow based on shadowgraph (Lin et al. 2002a) and PDPA measurements (Lin et al. 2004) are plotted simultaneously, for comparison. These correlations, which are among the most recent ones in this kind of flow topology with compressible crossflow are chosen because the experimental conditions are identical to this study except for the crossflow Mach number and because of the lack of experimental data for hypersonic crossflows. The correlations were obtained based on the least squares method, also used in the work of Gruber et al. (2000) and Leong et al. (2001) who have proposed similar expressions for penetration height. The expressions for the penetration height ' $h(x)$ ' as function of the spatial distance from the center of the orifice ' $x$ ' and orifice diameter ' $d_{j}$ ' measured with the shadowgraph and PDPA imaging techniques are, respectively $\frac{h}{d_{j}}=4.73 \times q^{0.3} \times\left(\frac{x}{d_{j}}\right)^{0.3}$

and

$\frac{h}{d_{j}}=3.94 \times q^{0.47} \times\left(\frac{x}{d_{j}}\right)^{0.21}$

Although these experimental profiles do not fit exactly with the experimental data, the tendencies are similar. For this reason, and also because the mentioned correlations are derived for compressible flows, a modification of Eqs. 2. and 3 is suggested in Eq. 4, which fits the Mach 6 experimental observations better (Fig. 13). The relative error between the correlations from literature and the proposed one varies in a range of $25-30 \%$ (especially in the region close to the injector, which might be critical for scramjet applications), justifying a new experimental fit for hypersonic crossflow condition. The profile for the $M=6$ experiments presents a less inclined slope for the jet trajectory, showing the higher pressure distribution exerted by the hypersonic crossflow on the jet surface compared to supersonic crossflows.
Fig. 12 a Standard deviation and $\mathbf{b}$ detected boundaries for $q=10$ jet $(1$ pixel $=0.42 \mathrm{~mm})$

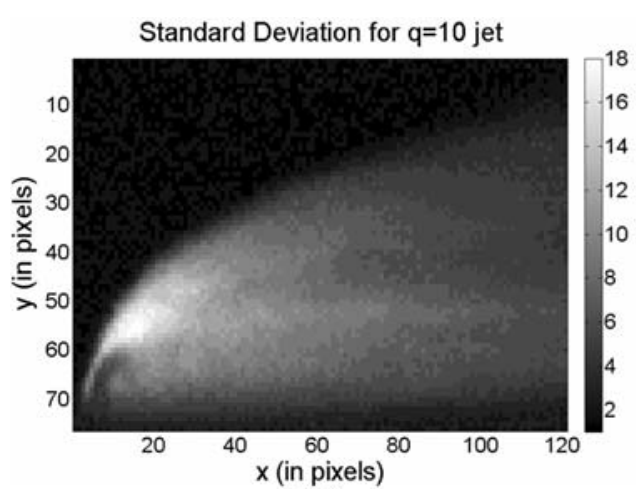

(a)

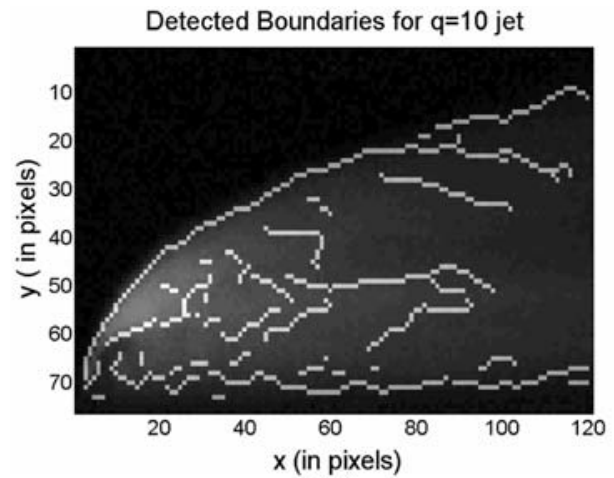

(b) 
Fig. 13 Penetration height versus downstream distance: $q=\mathbf{a} 4, \mathbf{b} 7$ and $\mathbf{c} 10$ jet

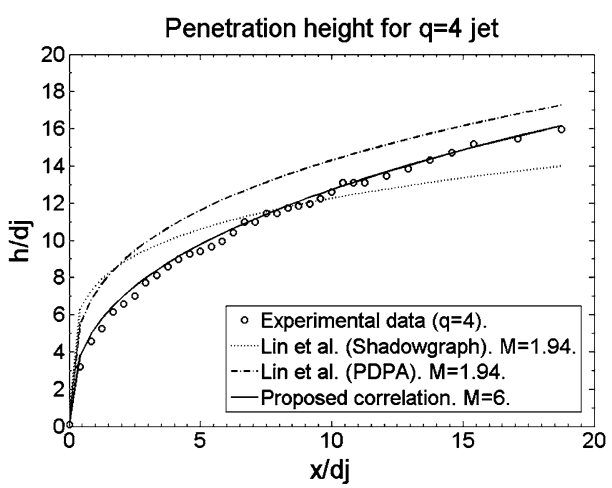

(a)

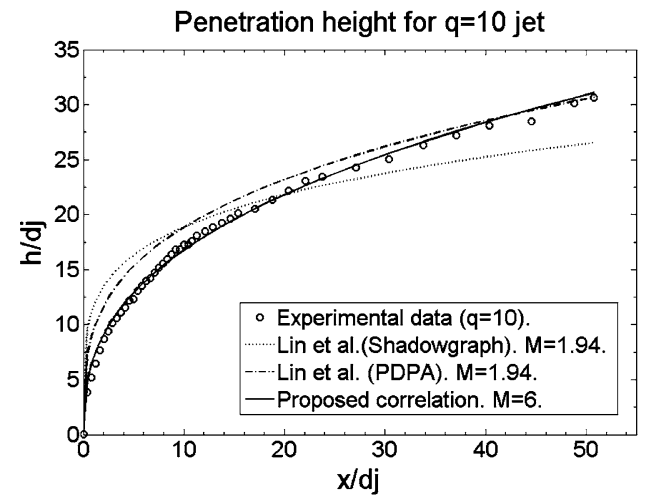

(c)

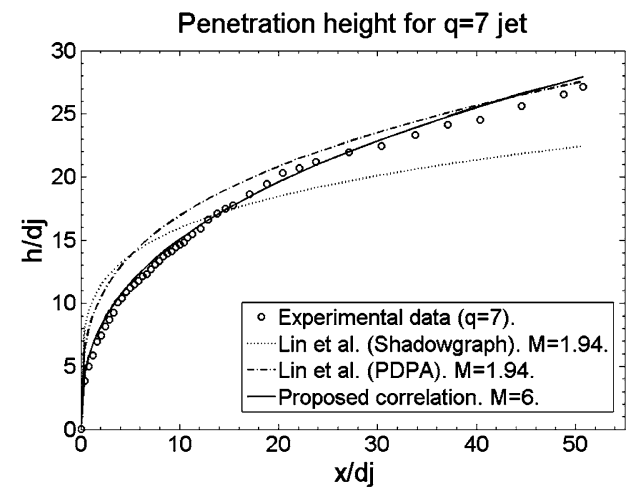

(b) $\frac{h}{d_{j}}=3.5 \times q^{0.3} \times\left(\frac{x}{d_{j}}\right)^{0.38}$

The evolution of the penetration height as a function of the injector ratio at a downstream distance of $5 \mathrm{~mm}$ for different shaped injectors (Fig. 14b) is shown in Fig. 14a. Jets emerging from the streamwise rectangular injector (aspect ratio $=1 / 3$ ) penetrates higher than the circular injector (aspect ratio $=1$ ), whereas the liquid penetrates to lower height in the case of spanwise rectangular injector (aspect ratio $=3$ ). The reason for this change in penetration height is that for the streamwise injector $(A R=1 / 3)$, the frontal (windward) is the smallest. The injected liquid is subjected to less momentum exchange with the free stream and thus exhibits a deeper penetration into the free stream. It can be concluded that the lift-off and the penetration height decreases as the aspect ratio increases as depicted in Fig. 14a. This change in penetration height is more pronounced for lower injection rates, as can be seen in Fig.14a, for a $q=1$ jet. These conclusions agree well with those presented by Haven and Kurosaka (1997). Although the results are presented at a constant distance $\left(x / d_{j}=5\right)$ as an example, similar results are obtained at different $x / d_{j}$ locations. As a result, spanwise rectangular injectors are more suitable for film cooling applications where the cooling fluid should be attached to the surface for low injection rates. A circular or streamwise rectangle injector on the other hand is more appropriate for fuel injection, where big penetration height and better mixing with crossflow are required.

Fig. 14 a Penetration height for different injectors and $\mathbf{b}$ trend of jet penetration with injector shape

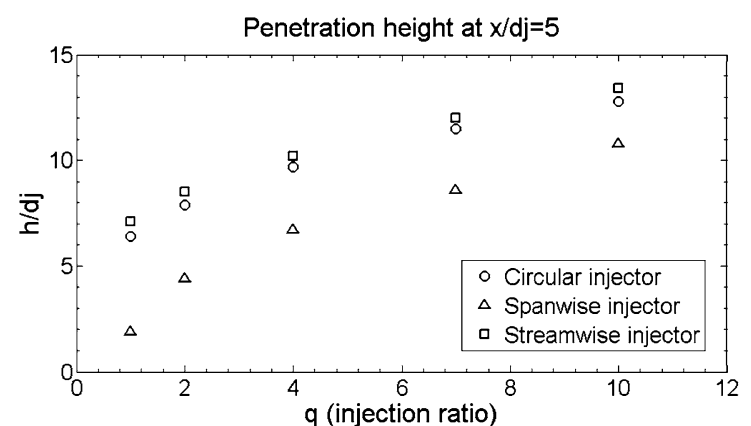

(a)

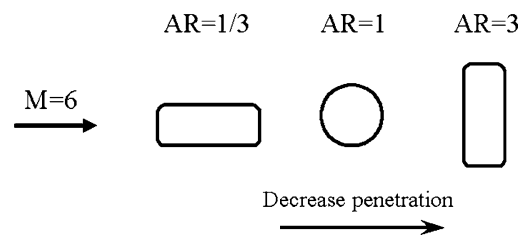

(b) 


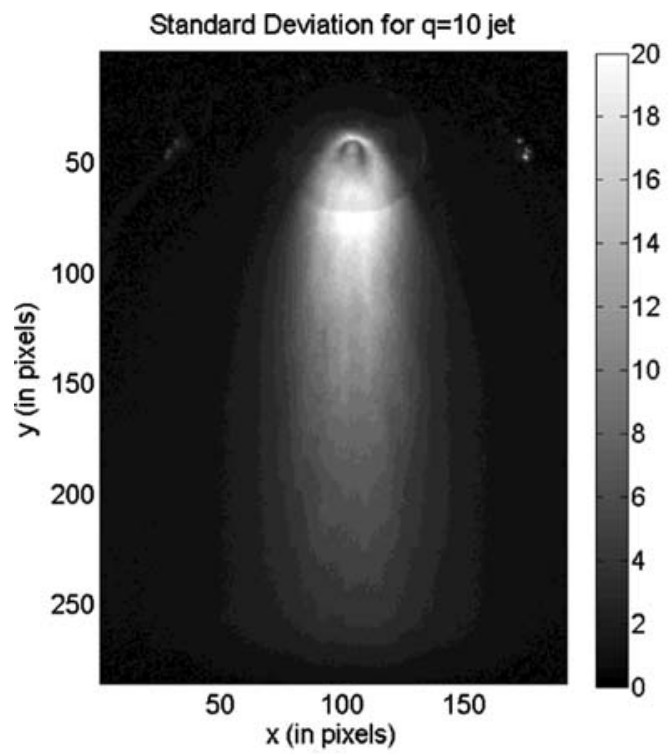

(a)

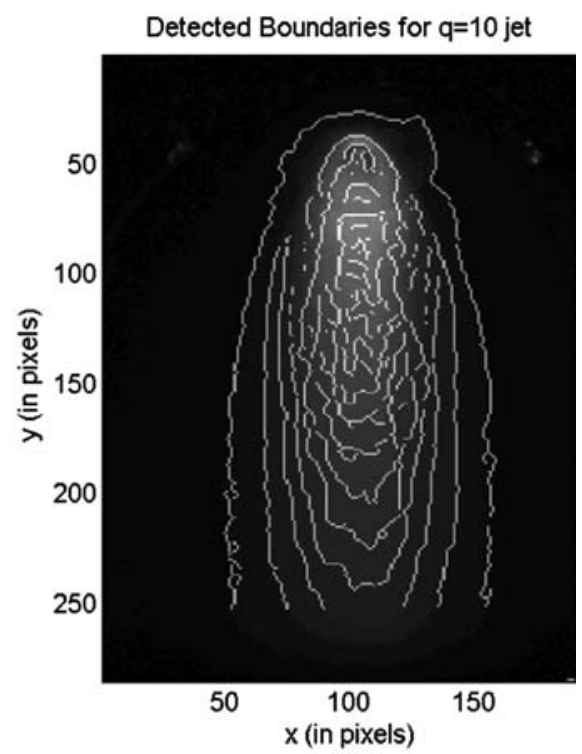

(b)

Fig. 15 a Standard deviation and $\mathbf{b}$ detected boundaries for $q=10$ jet $(1$ pixel $=0.33 \mathrm{~mm})$

\subsection{Lateral extension}

A similar threshold-based image binarization is applied as discussed in the previous section, followed by edge detection. The determination of the standard deviation is achieved by using 1,000 images. An example of the calculated standard deviation and the detected boundaries are shown in Fig. 15.

As for the penetration height, based on the least squares methods, an experimental fit is proposed for the lateral extension of circular jets. This is shown in Eq. 5 .

$\frac{w}{d_{j}}=2.1 \times q^{0.38} \times\left(\frac{x}{d_{j}}\right)^{2.47}$

Based on top view images, lateral extensions ' $w / d_{j}$ ' of the liquid jet are compared at $x=30 \mathrm{~mm}$ as an example, for the three injector shapes and various injection ratios (Fig. 16). The spanwise injector yields the widest lateral extension while the streamwise injector yields the lowest. For the spanwise injector ( $\mathrm{AR}=3$ ), the increase in the frontal (windward) surface of the liquid jet means more liquid can directly exchange momentum with the freestream air, resulting in a quicker bending of the jet to have a lower penetration height. As conclusion that can be derived from this analysis is that the lateral extension of the jet increases as the aspect ratio increases (Fig. 16b).

In agreement with the studies of Kolpin et al. (1968) and Joshi and Schetz (1975), the lateral extension of the jet depends less on the injection ratio than on the cross-sectional dimension of the injector and all injector profiles show an almost linear relation between lateral extension and injection rate (Fig. 16a).

\subsection{Mixing}

The investigation of mixing between liquid jet and free air stream was based on front-light illuminated recordings. In view of a liquid's higher efficiency in light scattering compared to air, low pixel intensities would correspond to pure air with high intensity regions identifying pure water. Mixing fractions could be inferred from the intensity scales. Messersmith and Dutton (1996) introduced the
Fig. 16 a Lateral extension for different injectors and $\mathbf{b}$ trend of jet lateral extension with injector shape

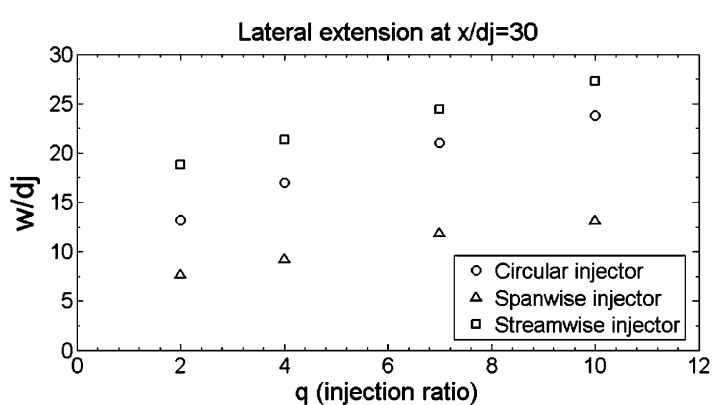

(a)

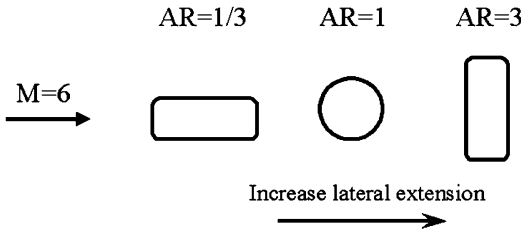

(b) 
Fig. 17 a Probability contour at $x / d_{j}=5$ and $\mathbf{b}$ top view

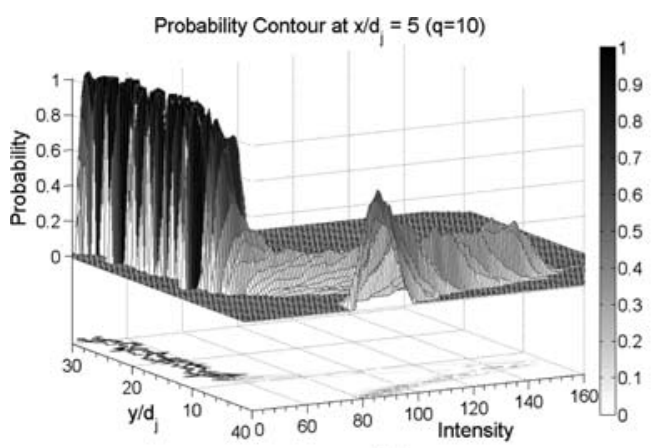

(a)

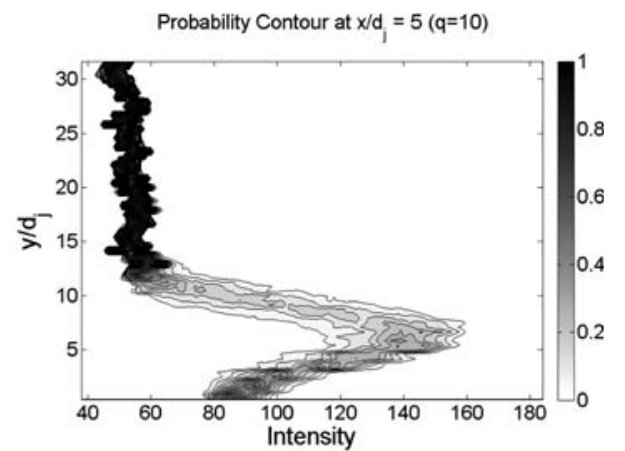

(b) concept of PDF based on image intensities in the identification of transport characteristics of mixing layers. They measured the probability of occurrence of all mixture fractions at various locations within the mixing layer demonstrating the validity of this tool in the study of waterair mixed phase-flows.

Based on 1000 consecutive images, the intensity probability distribution functions for pixel intensities were retrieved. Fig. 17 depicts the PDF together with a top view projection for a downstream location of $x / d_{j}=5$. At $y / d_{j}$ values exceeding 13 , i.e. above the jet boundary, the small width of the probability contour indicates the high probability of finding a low intensity signal (Fig. 17b). This corresponds to the small variation in background intensity which in itself attains low values. With decreasing $y / d_{j}$ the jet region is entered with an accompanying increase in intensity due to light-scattering of liquid droplets. For this case the sharp transition from low to high intensities facilitate determination of the jet's penetration height.

Probability contours at successive downstream locations for a $q=10$ jet case are presented in Fig. 18. For short downstream distances $\left(1 \leq x / d_{j} \leq 5\right)$, the small widths of the probability contours indicate inefficient mixing of the flow as a result of particles passing in large clumps. With increasing downstream distance $\left(9 \leq x / d_{j} \leq 12\right)$ the width of the contours increases. Intensity values have larger temporal fluctuations, indicating the presence of smaller clumps. These fluctuations increase further downstream $\left(12 \leq x / d_{j} \leq 20\right)$ showing that the formed clumps decompose into smaller droplets due to the action of surrounding aerodynamic forces of the crossflow. At $x / d_{j}=40$, full mixing between flow and liquid (meaning the disappearance of the liquid core) can be inferred from the smoothened transition from low to high intensity and a simultaneous enlarging of contour width. Far donwstream, at $x / d_{j}=100$, the intensity values decrease considerably and the probability of finding a low intensity signal is very high (practically 1), showing that water droplets are hardly captured by the camera. This fact could mean that the atomization process is complete and that no more droplet breakup happens. Lin et al. (2004) showed that completion of the liquid atomization process is reached after $x / d_{j}=100$. Anyhow, this technique does not allow to estimate accurately whether the atomization of the water particles has finished or not and additional measurements to detect the droplet diameter should be performed. Although not depicted, similar distances were witnessed before the occurrence of complete mixing for $q=4$ and 7 circular jets, respectively.

\subsection{Jet velocity}

To estimate the mean velocity field of the liquid jet particles recorded images were analysed by means of an in-house developed software based on the conventional PIV technique (Theunissen et al. 2006). For the current kind of application, small scale structures are no longer of interest. Instead, displacements of the larger more energetic structures require estimation. To retrieve robust velocity information from the succession of images, the analysis metrology was therefore based on the ensemble correlation approach (Meinhart et al. 2000).

As the detached clumps constitute detectable features within the recorded images, their motion can be traced by means of a cross-correlation operator between two successive images. Images are subdivided into so-called interrogation windows which are correlated. The resulting correlation map typically contains a dominant peak indicating the window-averaged displacement. This procedure is common within the PIV applications. However, PIV images typically consist of individual particle images whereas the intensity distributions in this case are continuous. In order to enhance the peak detectability within the correlation map, ensemble correlation is applied. The latter consists in averaging the individual correlation functions prior to analysis of the correlation map. To alleviate the degrading influence of spatial gradients in the reigning velocity field, images are iteratively deformed, such that the image features are better followed. Because the retrieved displacement vector represents a spatially filtered version of the underlying velocity 


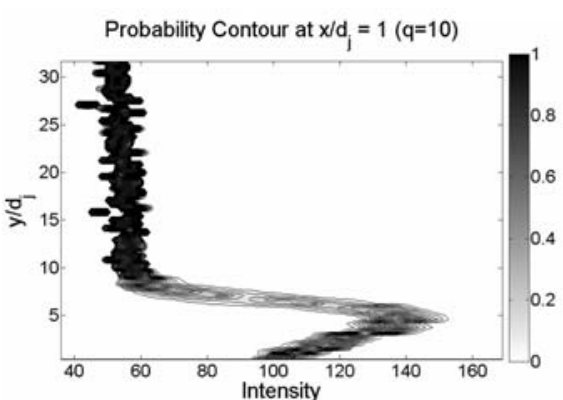

(a)

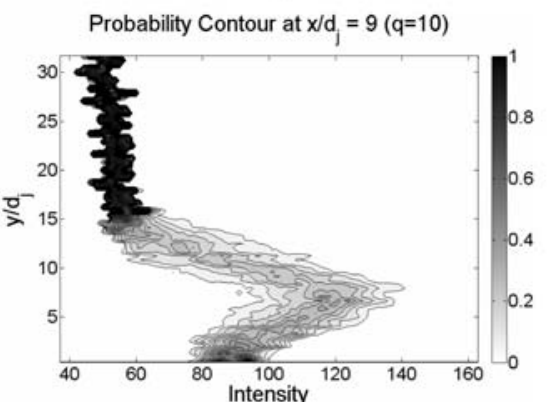

(d)

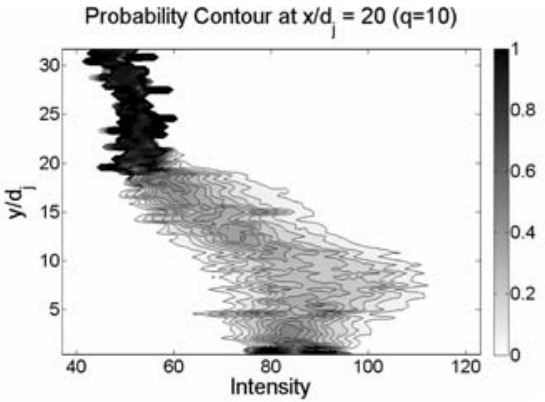

(g)

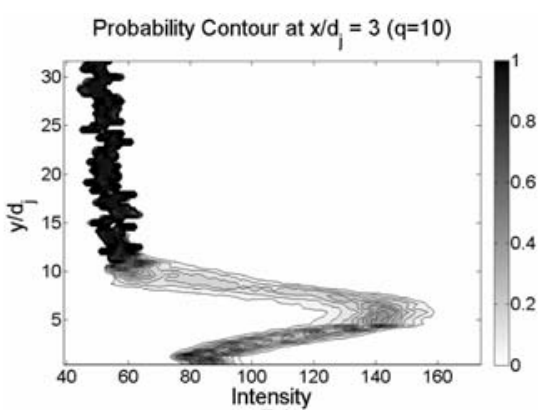

(b)

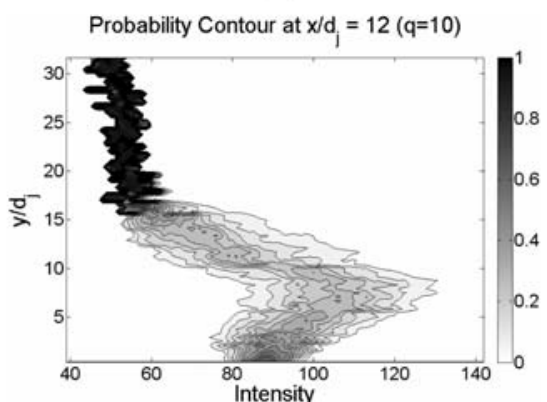

(e)

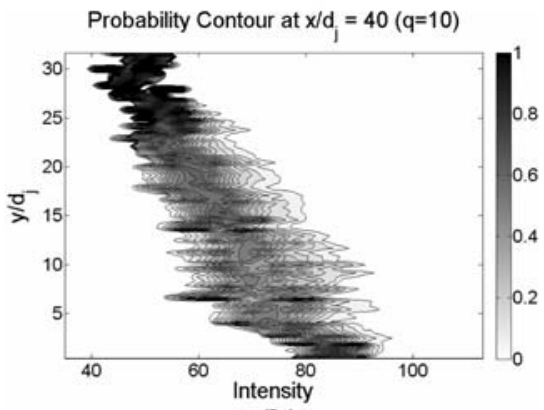

(h)

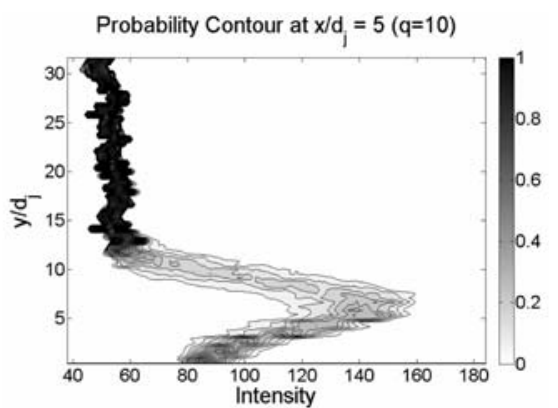

(c)

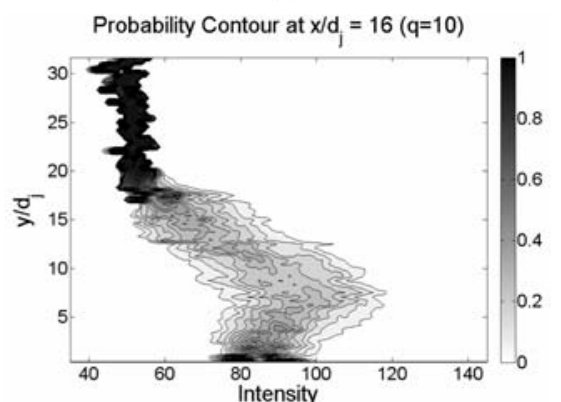

(f)

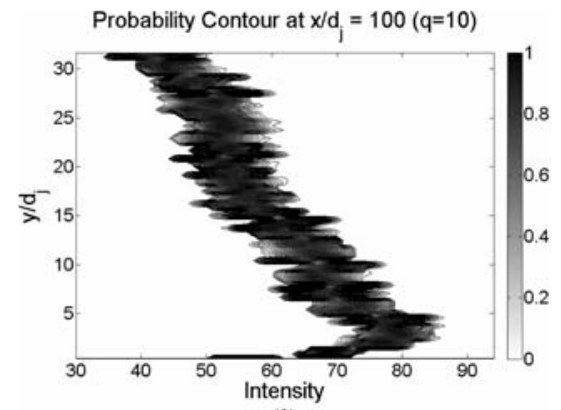

(i)

Fig. 18 Probability contours for successive downstream locations. $x / d_{j}=\mathbf{a} 1$, b 3, c 5, d 9, e 12, f 16, g $20, \mathbf{h} 40$ and i 100

field, smaller interrogation windows allow a reduced modulation effect. To obtain sufficient spatial resolution, iterative window deformation and refinement (Scarano and Riethmuller 1999) were incorporated. Final window sizes of $17 \times 17$ pixels $\left(1.37 \times 1.37 \mathrm{~mm}^{2}\right)$ were applied in the analysis with an overlap factor of around $80 \%$ yielding a vector spacing of 3 pixels $(0.24 \mathrm{~mm})$. In total, 500 image couples were considered. Results presented hereafter refer to the case of a streamwise rectangular injector $(\mathrm{AR}=1 / 3)$, with a jet/air momentum-flux ratio of 7 . The use of backlight illumination extended the measurement volume perpendicular to the image plane. As such, objects moving perpendicular to the field of view were captured as well, degrading the image quality due to blurring and decreasing the level of accuracy in the image analysis. As the ensemblePIV technique is used for a dimensional analysis to predict the order of magnitude of the droplets velocity, no error estimation is performed. Nevertheless, it can be noted that the error in the estimation of in-plane velocity would be within $\pm 5 \%$, due to an out-of-plane velocity component of $10 \%$. However, for this type of injector most of the displacement has been shown to be concentrated in the bulk flow direction (Figs. 14 and 16), reducing such inaccuracies. This is also advocated by the sufficiently high signal-to-noise ratios $(\mathrm{SN}>4)$ in the leeward part of the jet, where the speed of the water droplets is lower (Fig. 19a). This ratio is a measure of the correlation quality (Keane and Adrian 1992) indicating a limited number of erroneous displacements when exceeding a level of 1.5. In regions with strong temporal variation in image quality, i.e. at the windward region of the jet where detachment and bursting of clumps take place, a degradation in $\mathrm{SN}$ ratios is noticeable but the levels remain acceptable $(\mathrm{SN}>3)$.

The velocity vector field, superimposed onto contours of the horizontal velocity is depicted in Fig. 19b. High values of the velocity in the windward region of the jet show the rapid acceleration of the jet due to the aerodynamic forces of the crossflow. In the leeward region the velocity of the 

circular injector at $q=7$. a Signal noise ratio; $\mathbf{b}$ ensemble average velocity vectors superimposed onto average contours of horizontal velocity component
Fig. 19 PIV measurement for

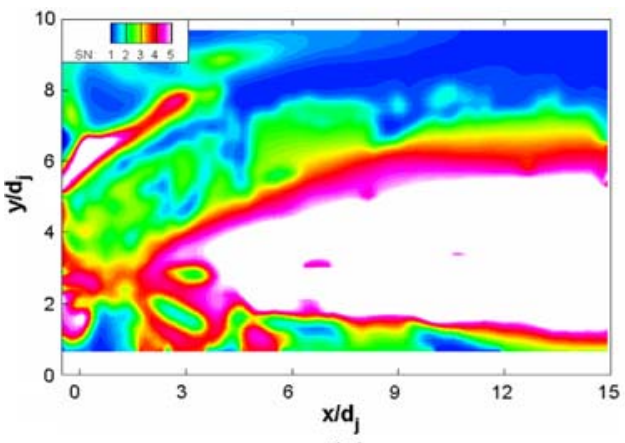

(a)

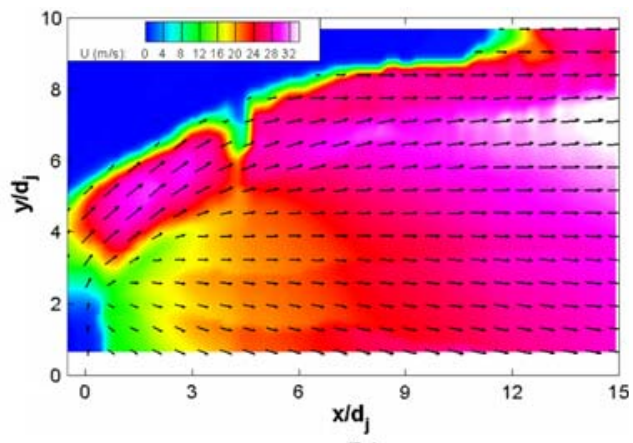

(b) disintegrated small droplets is smaller whilst they accelerate progressively. The image analysis predicts a vertical velocity of around $20 \mathrm{~m} / \mathrm{s}$ at the jet injection point thus matching the jet's exit velocity measured with the rotameter. At a downstream distance of 16 injector diameters, the disintegrated small droplets in the leeward region reached a velocity of $40 \mathrm{~m} / \mathrm{s}$ (Fig. 19b). The high velocity of the detached clumps in the windward region is not well captured as it can be inferred from the lower SN ratio value. Downstream of the strong bow shock, an air velocity of the crossflow in the order of $160 \mathrm{~m} / \mathrm{s}$ can be expected from the normal shock relations. The measured particle velocities in the leeward region of the jet are lower, meaning that larger downstream distances are required for the water particles in this region to accelerate up to the free stream velocity.

Figure 20 shows stream traces plotted onto the ensemble averaged intensities of 500 images for the case of the streamwise rectangular injector jet with $q=7$. Deflection of the jet due to the high pressure distribution on its surface above the injection point is noticeable. The liquid jet is diverted from vertical to horizontal direction. At about 10 injector diameters downstream of the injection, the vertical component has decreased to negligible values. The latter observation agrees with the evolution of the horizontal velocity (Fig. 19b).

\section{Frequency analysis}

A frequency analysis of the clump detachment during the jet breakup and of the fluctuating shock pattern has been carried out by means of Fast Fourier Transforms on the times series of recorded pixel intensities. Power spectral densities were calculated by the method of Welch (1967).

\subsection{Jet breakup and clump detachment}

As explained, growing axial waves lead to the primary breakup of the jet through clump detachment along the

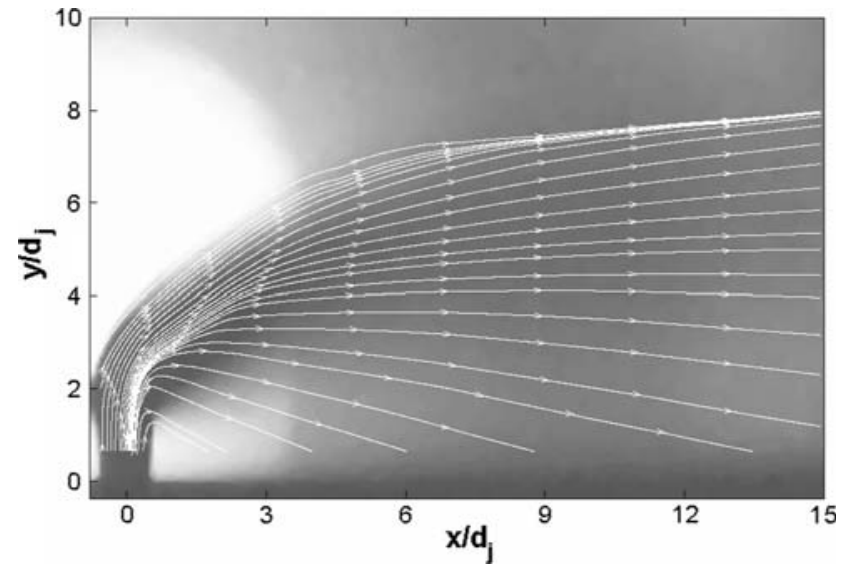

Fig. 20 Stream traces of the liquid jet, after ensemble-PIV analysis

windward region of the jet and this tear of clumps is influenced by the bow shock-separation shock interaction. The liquid fragmentation is expected to happen with a certain frequency, deserving a frequency analysis of the phenomena. In Fig. 21a, an instantaneous image of a back-lighted $q=10$ circular injector jet case is shown. Frequency spectra of pixel intensities corresponding to different regions of the jet breakup are expected to indicate the existence of characteristic frequencies. Power spectral densities are plotted for representative pixels of three distinct regions in the frequency map in Fig. 21b-d. Pixel intensities in the windward part of the jet (1) are observed to possess a dominant frequency of $4.5 \mathrm{kHz}$ which is related with the clump detachment. A Strouhal number can be defined based on the jet diameter and the jet exit velocity $S t=\frac{f \times d_{j}}{U_{j}}$. With this definition of the velocity scale, a Strouhal number of $S t=0.18$ is obtained for the clump detachment and breakup of the liquid jet. The leeward region (2) is not in direct contact with the high speed crossflow and the clump fracture noticed in the windward part is absent. Instead, the jet disintegrates in small particles at a lower frequency of $1.5 \mathrm{kHz}$. In the free stream (3), no dominant frequency is apparent except for a $50 \mathrm{~Hz}$ peak attributed to electrical noise. 
Fig. 21 a Instantaneous image of liquid jet breakup and power spectral densities for pixels in b windward region, c leeward region and $\mathbf{d}$ free stream of $q=10$ circular jet

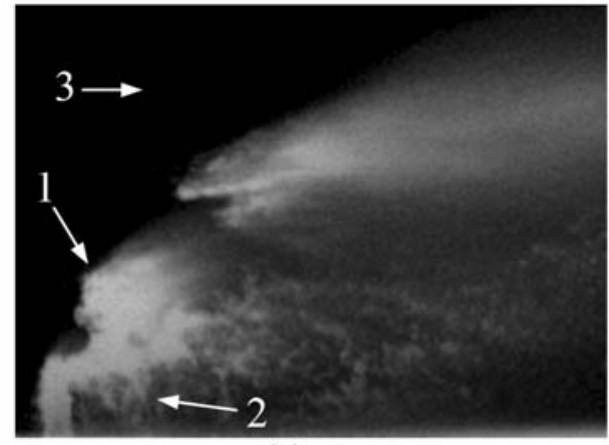

(a)

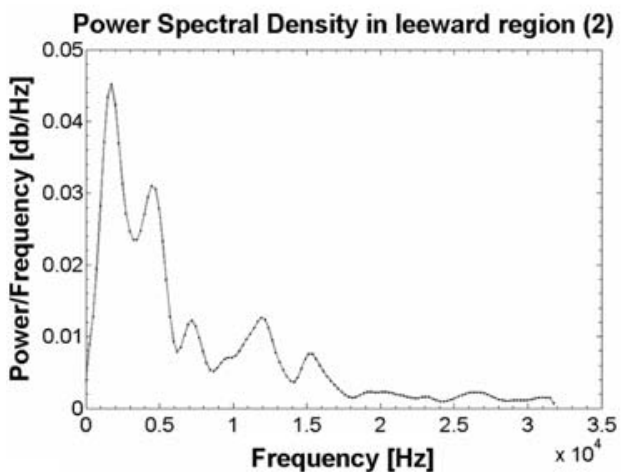

(c)

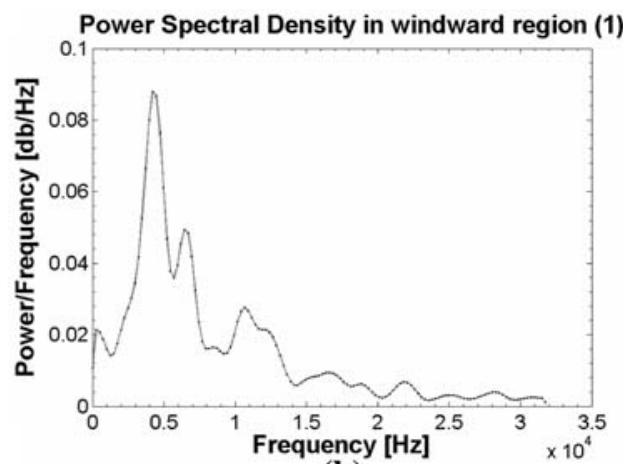

(b)

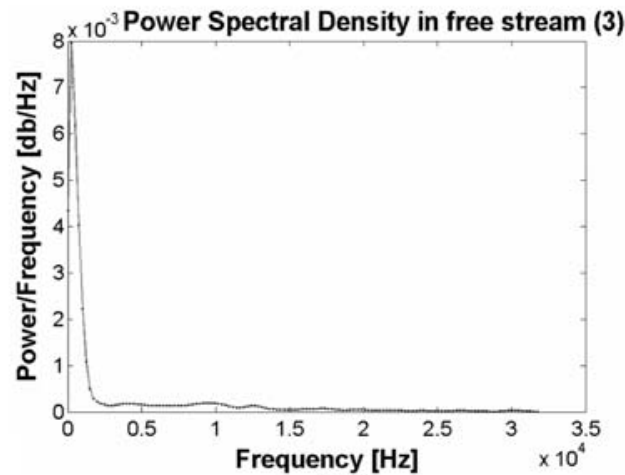

(d)
Concluding, clump detachment within the windward region is responsible for the primary breakup of the jet and occurs with a specific frequency of $4.5 \mathrm{kHz}$ and
$S t=0.18$. Almost identical frequencies are found for lower injection rates of the circular jet $(q=4$ and 7 , respectively).
Fig. 22 a Standard deviation of 1,000 images and frequency spectrums for pixels in (a) separation shock, b bow shock and $\mathbf{c}$ free stream of $q=10$ circular jet

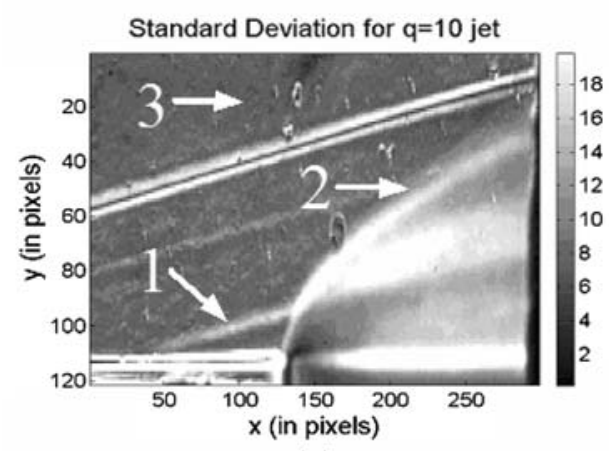

(a)

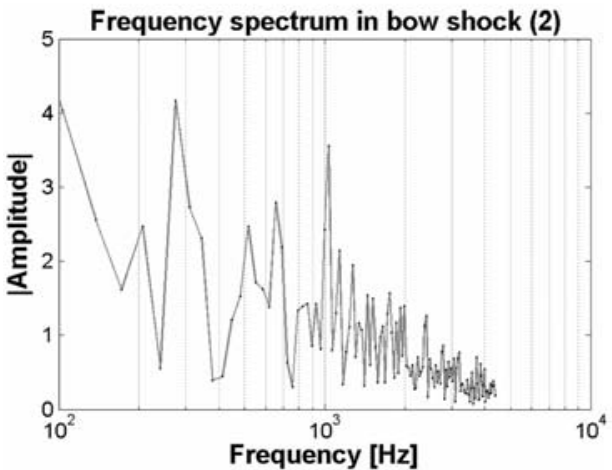

(c)

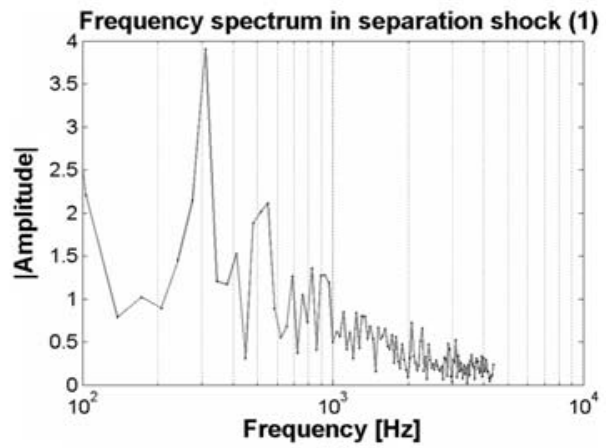

(b)

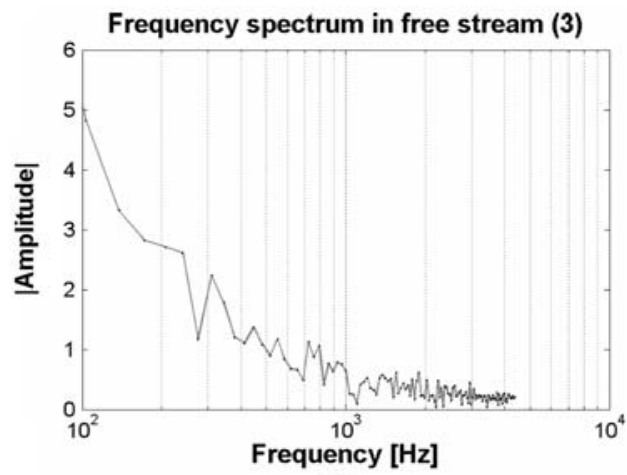

(d) 


\subsection{Fluctuating shock pattern}

The whipping phenomenon is related with the interaction between the bow shock and separation shock and the impulse of the liquid jet and, as a consequence, a continuous vibration of the shock system. This phenomenon has not been reported in previous studies (or the authors have not obtained a valid reference) and needs to be investigated further. Figure 22 visualizes the standard deviation of the intensity signal for 1,000 Schlieren images. High amplitudes in RMS are observed to occur in the flow domain occupied by the fluctuating bow shock and separation shock. In the region spanned by the separation shock region (1), a dominant frequency in pixel intensity appears around $300 \mathrm{~Hz}$. A Strouhal number can be defined based on the length of the separation shock (from the separation point to the impingement point of the separation shock on the bow shock) and the difference in velocity between the crossflow velocity and the jet exit velocity $S t=\frac{f \times d}{\left(U_{\infty}-U_{j}\right)}$. Cortelezzi and Karagozian (2001) explained in their study of the counter rotating vortex pair formation that either the jet velocity or the crossflow velocity may not be an appropriate velocity scale since the dynamics of the rollup of the shear layer are dependent of both velocities. Based on this velocity scale, a Strouhal number of $S t=0.011$ is obtained for the separation shock oscillation. In the bow shock region (2), in addition to the peak at $300 \mathrm{~Hz}$, higher frequency peaks are present at $1 \mathrm{kHz}$ and above (note that the peak at $100 \mathrm{~Hz}$ originates from the electrical noise peak at $50 \mathrm{~Hz}$ ). These peaks indicate that the bow shock oscillation may be related with the high frequency clump detachment on the windward region of the jet. In the free stream (3), no dominant frequency is observed apart from the electrical noise peak. Frequencies in the same order of magnitude of $300-400 \mathrm{~Hz}$ for the separation shock fluctuation are found for different injection ratios $(q=2-10)$.

\section{Conclusions}

Liquid jet injection into Mach 6 hypersonic flow is investigated experimentally by a high-speed camera and by employing different image processing techniques. Water is injected at different momentum-flux ratios through different injector shapes having different aspect ratios. Correlations for the penetration height and lateral extension are proposed relating the injection momentum ratio and orifice diameter. Comparison of penetration heights for different injectors at certain downstream locations revealed that penetration height decreases as the injector's aspect ratio increases. In the same way, the lateral extension of the jet increases proportionally with the increasing aspect ratio of the injector. Injectors with higher aspect ratios are therefore more suitable for film cooling applications, e.g. for the cooling of combustion chambers in scramjets or for active thermal protection systems of space vehicles. On the other hand, streamwise rectangular and circular injectors with low aspect ratios are more suitable for fuel injection in scramjet applications. Mixing of the jet with the surrounding hypersonic crossflow is shown to be complete at a distance of $x / d_{j} \sim 40$, independent of the momentum-flux ratio. The fracture of clumps for a circular $q=10$ jet appears at a frequency of $4.5 \mathrm{kHz}$ and $S t=0.18$ based on the jet diameter and velocity. Similar values are obtained for lower injection rates and other injectors. This liquid fracture can be influenced by the bow shock-separation shock interaction. The separation shock fluctuates at a frequency of $300-400 \mathrm{~Hz}$ and $S t=0.011$ (based on the crossflow and jet velocities difference and the separation shock length) independent of the injection ratio. Higher frequencies are found for the bow shock fluctuation $(1 \mathrm{kHz}$ and more). This indicates a probable coupling between these two phenomena of liquid clumps' detachment and shock system fluctuation.

Acknowledgments The authors wish to thank the French MOD, DGA, who provided the support to carry out this work in the framework of a research contract (Marché $\mathrm{N}^{\circ}$.06.70.101.00.470.75.96). A. Marangoz and V. Van der Haegen are kindly acknowledged for their helpful contributions.

\section{References}

Catton I, Hill DE, McRae RP (1968) Study of liquid jet penetration in a hypersonic stream. AIAA J 6(11):2084-2089

Cortelezzi L, Karagozian AR (2001) On the formation of the counterrotating vortex pair in transverse jets. J Fluid Mech 446:347-373

Delery J (1989) Shock/shock and shock-wave/boundary-layer interactions in hypersonic flows. AGARD Report 761(part 9)

Ghenai C, Sapmaz H, Lin CX (2005) Characterization of aerated liquid jet in subsonic and supersonic crossflow. AIAA-20053580, AIAA/ASME/SAE/ASEE joint propulsion conference and exhibit, 41st, Tucson, July 2005

Gruber MR, Nejad AS, Chen TH, Dutton JC (1996) Bow shock/jet interaction in compressible transverse injection flowfields. AIAA J 34(10):2191-2193

Gruber MR, Nejad AS, Chen TH, Dutton JC (2000) Transverse injection from circular and elliptic nozzles into a supersonic crossflow. J Propul Power 16(3):449-457

Fric TF, Roshko A (1994) Vortical structure in the wake of a transverse jet. J Fluid Mech 279:1-47

Haven BA, Kurosaka M (1997) Kidney and anti-kidney vortices in crossflow jets. J Fluid Mech 352:27-64

Hinson WF, Gooderum PB, Bushnll DM (1970) Experimental investigation of multiple-jet liquid injection into hypersonic flow. NASA TN D-5861

Joshi BP, Schetz JA (1975) Effect of injector shape on penetration and spread of liquid jets. AIAA J 13(9):1137-1138

Keane RD, Adrian RJ (1992) Theory of cross-correlation analysis of PIV images. App Sci Res 49:191-215 
Kolpin MA, Horn KP, Reichenbach RE (1968) Study of penetration of a liquid injectant into a supersonic flow. AIAA J 6(5):13851390

Krothapalli A, Lourenco L, Buchlin JM (1989) On the separated flow upstream of a jet in a crossflow. AIAA-89-0571

Leong MY, McDonell VG, Samuelsen GS (2001) Effect of ambient pressure on an airblast spray injected into a crossflow. J Propul Power 17(5): 1076-1084

Less DM, Schetz JA (1986) Transient behavior of liquid jets injected normal to a high velocity gas stream. AIAA J 24(12):1979-1986

Li HS, Karagozian AR (1992) Breakup of a liquid jet in supersonic crossflow. AIAA J 30(7):1919-1921

Lin K-C, Kennedy PJ, Jackson TA (2002a) Penetration heights of liquid jets in high-speed crossflows. AIAA-2002-0873, AIAA aerospace sciences meeting and exhibit, 40th, Reno, 14-17 January 2002

Lin K-C, Kennedy PJ, Jackson TA (2002b) Structures of aeratedliquid jets in high-speed crossflows. AIAA-2002-3178, AIAA fluid dynamics conference and exhibit, 32nd, St. Louis, 24-26 June 2002

Lin K-C, Kennedy PJ, Jackson TA (2004) Structures of water jets in a mach 1.94 supersonic crossflow. AIAA-2004-0971, AIAA aerospace sciences meeting and exhibit, 42nd, Reno, 5-8 January 2004

Marangoz A, Chazot O, Asma CO (2006) Investigation of liquid jet injection into a hypersonic crossflow. Project Report 2006-21, von Karman Institute for Fluid Dynamics, Brussels

Meinhart CD, Werely ST, Santiago JG (2000) A PIV algorithm for estimating time-averaged velocity fields. J Fluids Eng 122:285289

Messersmith NL, Dutton JC (1996) Mie scattering measurements of probability density functions in compressible mixing layer. Exp Fluids 21:291-301
Muraggapan S, Gutmak E, Carter C (2005) Control of penetration and mixing of an excited supersonic jet into a supersonic crossstream. AIAA 2005-612, P AIAA Aerospace Sciences Meeting and Exhibit, 43rd, Reno, January 2005

Nejad AS, Schetz JA (1984) Effects of viscosity and surface tension on a jet plume in supersonic crossflow. AIAA J 22(4):458-459

Reichenbach RE, Horn KP (1971) Investigation of injectant properties on jet penetration in a supersonic stream. AIAA J 9(3):469472

Sallam KA, Aalburg C, Faeth GM (2004) Breakup of round nonturbulent liquid jets in gaseous crossflow. AIAA J 42(12): 2529-2540

Scarano F, Riethmuller ML (1999) Iterative multigrid approach in PIV image processing with discrete window offset. Exp Fluids 26:513-523

Schetz JA, Kush EA Jr, Joshi PB (1980) Wave phenomena in liquid jet breakup in a supersonic crossflow. AIAA J 18(7):774-778

Theunissen R, Buchmann N, Corieri P, Riethmuller ML, Darquenne C (2006) Experimental investigation of aerosol deposition in alveolar lung airways. In: 13th International symposium on applications of laser techniques to fluid mechanics, Lisbon

Welch PD (1967) The use of fast fourier transform for the estimation of power spectra: a method based on time averaging over short, modified periodgrams. IEEE Trans Audio Electract 15(2):70-73

Yakar B, Hanson RK (1998) Experimental investigation of flame holding capability of a transverse hydrogen jet in supersonic crossflow. In: International symposium on combustion, 27th, Boulder, pp 2173-2180 